\title{
Research Article \\ On Complementary Root Locus of Biproper Transfer Functions
}

\author{
Marcelo C. M. Teixeira, ${ }^{1}$ Edvaldo Assunção, ${ }^{1}$ Rodrigo Cardim, ${ }^{1}$ \\ Neusa A. P. Silva, ${ }^{2}$ and Erica R. M. D. Machado ${ }^{2}$ \\ ${ }^{1}$ Department of Electrical Engineering (DEE), Faculdade de Engenharia de Ilha Solteira, \\ UNESP—São Paulo State University, 15385-000 Ilha Solteira, SP, Brazil \\ ${ }^{2}$ Department of Mathematics (DM), Faculdade de Engenharia de Ilha Solteira, \\ UNESP_São Paulo State University, 15385-000 Ilha Solteira, SP, Brazil
}

Correspondence should be addressed to Marcelo C. M. Teixeira, marcelo@dee.feis.unesp.br

Received 12 February 2009; Revised 22 May 2009; Accepted 11 August 2009

Recommended by J. Rodellar

This paper addresses the root locus (locus of positive gain) and the complementary root locus (locus of negative gain) of biproper transfer functions (transfer functions with the same number of poles and zeros). It is shown that the root locus and complementary root locus of a biproper transfer function can be directly obtained from the plot of a suitable strictly proper transfer function (transfer function with more poles than zeros). There exists a lack of sources on the complementary root locus plots. The proposed procedure avoids the problems pointed out by Eydgahi and Ghavamzadeh, is a new method to plot complementary root locus of biproper transfer functions, and offers a better comprehension on this subject. It also extends to biproper open-loop transfer functions, previous results about the exact plot of the complementary root locus using only the well-known root locus rules.

Copyright (c) 2009 Marcelo C. M. Teixeira et al. This is an open access article distributed under the Creative Commons Attribution License, which permits unrestricted use, distribution, and reproduction in any medium, provided the original work is properly cited.

\section{Introduction}

The analysis of the location of the closed-loop poles in the s-plane and in function of a positive gain in the open-loop transfer function, proposed by Evans [1,2], has found wide application in the analysis and design of linear time-invariant systems. Recently, the root locus method was extended to fractional-order systems [3,4]. More details about the design of controllers based on root-locus method or depending on one parameter can be found in [5-12]. The term complementary root locus, that means locus of the closed-loop poles in function of a negative open-loop gain, was proposed by Narendra, considering that the root locus and the complementary root locus together form a complete algebraic curve [13]. 
In the design of control systems, for changing time constants, and when large loop gain is required in inner loops, the complementary root locus is useful tool [14]. Further results about the complementary root locus can be found in [14].

There exists a lack of sources on the complementary root locus and many textbooks about control systems present the root-locus method and its application in control design, but do not present information about complementary root locus $[15,16]$. The textbooks $[17-$ 21] present both methods.

In [14], the authors have shown that some of the steps in the method of constructing complementary root locus (construction rules) are not valid for open-loop transfer functions with the same number of poles and zeros, referred to as biproper transfer functions. Considering this fact, this problem was studied and new definitions and analyses were presented in [14] to plot the complementary root locus. This fact has motivated researches to find new methods to avoid these problems and to offer a better comprehension for the designers about this subject.

For instance, in [22] a method to plot complementary root locus using only the wellknown root-locus construction rules to plot root locus was proposed. This new technique allows an exact plot when the sum of the number of poles and zeros is odd and an approximated plot otherwise. An example in that paper illustrated the application of this method to approximately plot the complementary root locus of biproper transfer functions.

In this paper an alternative procedure to plot the complementary root locus of transfer functions that are biproper is presented. The main idea was to show that the root locus and complementary root locus of a biproper transfer function can be directly and exactly obtained from the plot of the root locus and complementary root locus of a suitable strictly proper transfer function (transfer function with more poles than zeros). This procedure removes the problem cited above, because the plots are done only with strictly proper open-loop transfer functions. The proposed method extends the results presented in [22], because it can also allow an exact plot of the complementary root locus of biproper transfer functions, using the well-known root-locus construction rules. An example illustrates this fact. Literature did not present many papers about the complementary root locus, and this paper offers a better comprehension on this subject.

\section{Statement of the Problem}

Given the feedback system described in Figure 1, consider that the open-loop transfer function is biproper:

$$
G(s) H(s)=\frac{b_{n} s^{n}+b_{n-1} s^{n-1}+\cdots+b_{1} s+b_{0}}{s^{n}+a_{n-1} s^{n-1}+\cdots+a_{1} s+a_{0}},
$$

where $K_{0}, b_{n}, b_{i}, a_{i} \in \mathbb{R}$, for $i=0, \ldots, n-1$ and $b_{n} \neq 0$.

Now, define

$$
K=K_{0} b_{n} .
$$

Then, draw the root locus and complementary root locus of the closed-loop system given in (2.1) and Figure 1 for $-\infty<K<\infty$, using only the plots of the root locus and complementary root locus of an equivalent system with a strictly proper open-loop transfer function. 


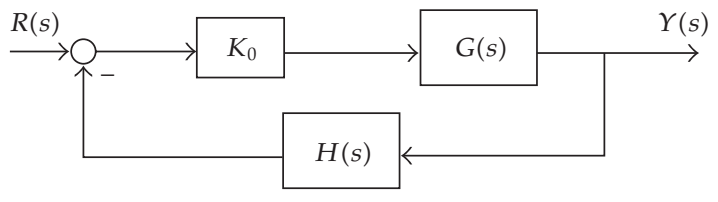

Figure 1: A closed-loop controlled system.

\section{Root Locus and Complementary Root Locus of Biproper Transfer Functions}

In this section a solution of the problem stated in Section 2 is presented.

From (2.1), (2.2), and Figure 1, the closed-loop transfer function is

$$
\begin{aligned}
\frac{Y(s)}{R(s)} & =\frac{K_{0} G(s)}{1+K_{0} G(s) H(s)} \\
& =\frac{K\left(s^{n}+c_{n-1} s^{n-1}+\cdots+c_{1} s+c_{0}\right)}{K\left(s^{n}+c_{n-1} s^{n-1}+\cdots+c_{1} s+c_{0}\right)+\left(s^{n}+a_{n-1} s^{n-1}+\cdots+a_{1} s+a_{0}\right)},
\end{aligned}
$$

where $c_{i}=b_{i} / b_{n}, i=0,1, \ldots, n-1$.

Therefore, the characteristic equation of this feedback system is

$$
\begin{aligned}
\Delta(s) & =K\left(s^{n}+c_{n-1} s^{n-1}+\cdots+c_{1} s+c_{0}\right)+\left(s^{n}+a_{n-1} s^{n-1}+\cdots+a_{1} s+a_{0}\right) \\
& =K N(s)+D(s)=0,
\end{aligned}
$$

where $N(s)=\left(s^{n}+c_{n-1} s^{n-1}+\cdots+c_{1} s+c_{0}\right)$ and $D(s)=\left(s^{n}+a_{n-1} s^{n-1}+\cdots+a_{1} s+a_{0}\right)$.

Note that if $K$ defined in (2.2) is positive, then one can directly apply the classical root-locus construction rules to plot the root locus of (3.2). The reader can find these rules, for instance, in $[15,16,18-20,23]$.

In the case where $K<0$, Eydgahi and Ghavamzadeh [14] showed that one cannot directly apply the classical rules to plot the complementary root locus. It is necessary to consider additional properties, related to the roots at infinity [14]. In this case, a method presented in [22] allows an approximated plot, using only the root-locus construction rules.

The next theorem offers an alternative method to solve the problem stated in Section 2, without the approximated plot of [22] or the additional definitions and analyses presented in [14]. It also complements the available results and offers a better understanding about this subject.

Theorem 3.1. The root-locus and complementary root-locus plots $(-\infty<K<\infty)$ of the system given in Figure 1 with the characteristic equation (3.2), whose open-loop transfer function is biproper, can be directly obtained from the root-locus and complementary root-locus plots of the strictly proper open-loop system $\widetilde{K} \widetilde{N}(s) / D(s)$ given by its characteristic equation:

$$
\widetilde{K} \widetilde{N}(s)+D(s)=0,
$$


where

$$
\begin{gathered}
D(s)=s^{n}+a_{n-1} s^{n-1}+\cdots+a_{1} s+a_{0}, \quad N(s)=s^{n}+c_{n-1} s^{n-1}+\cdots+c_{1} s+c_{0}, \\
\widetilde{N}(s)=N(s)-D(s)=\left(c_{n-1}-a_{n-1}\right) s^{n-1}+\left(c_{n-2}-a_{n-2}\right) s^{n-2}+\cdots+\left(c_{0}-a_{0}\right), \\
\widetilde{K}=\frac{K}{K+1} .
\end{gathered}
$$

The gain $\mathrm{K}$ can be obtained from the gain $\widetilde{K}$, using the following expression:

$$
K=\frac{\tilde{K}}{1-\tilde{K}} .
$$

Proof. From (3.2) and (3.5) it follows that

$$
K N(s)+D(s)=(K+1) N(s)+D(s)-N(s)=(K+1) N(s)-\widetilde{N}(s)=0 .
$$

Now, considering $K \neq-1$, then, from (3.8),

$$
N(s)-\frac{1}{K+1} \widetilde{N}(s)=0
$$

Again from (3.8), note that $K N(s)=-D(s)$. So, multiplying both sides of (3.9) by $K$ one obtains

$$
K N(s)-\frac{K}{K+1} \widetilde{N}(s)=-D(s)-\widetilde{K} \widetilde{N}(s)=0,
$$

where $\widetilde{K}$ was defined in (3.6). Then, (3.3) follows from (3.10). Now, note that (3.6) and (3.7) are equivalent. So, (3.7) can be used to calculate the gain $K$, given a gain $\widetilde{K}$. Finally, from (3.4) and (3.5), observe that the transfer $\widetilde{N}(s) / D(s)$ has more poles than zeros and so it is strictly proper.

Remark 3.2. The proof of Theorem 3.1 can also be done using block diagram manipulations, as described in Figure 2. Observe that in Figure 2, from (3.5), the block diagrams (b) and (c) are equivalent. The block diagrams are very useful in the analysis and design of control systems [15-21]. A systematic procedure for reducing block diagrams and its implementation in MATLAB program can be found in [24].

Remark 3.3. Figure 3 describes the relation between $K$ and $\tilde{K}$, from (3.7). Note that: (i) for $\widetilde{K}$ in the interval $-\infty<\widetilde{K}<\infty$, then $K$ presents also values in all interval $-\infty<K<\infty$; (ii) $K \rightarrow \infty$ when $\widetilde{K} \rightarrow 1^{-}$, and (iii) $K \rightarrow-\infty$ when $\tilde{K} \rightarrow 1^{+}$. Therefore, the root-locus plot $(0 \leq \widetilde{K}<\infty)$ and the complementary root locus $(-\infty<\tilde{K} \leq 0)$ of (3.3) correspond to the complete root loci plots $(-\infty<K<\infty)$ of the original equation (3.2). From Figure 3 one can see that the complementary root locus $(-\infty<K \leq 0)$ of (3.2) can be obtained from the root-loci plots of (3.3) for $-\infty<\tilde{K} \leq 0$ and $1<\tilde{K}<\infty$ can be obtained and the root locus $(0 \leq K<\infty)$ of (3.2) from the root locus of (3.3) for $0 \leq \tilde{K}<1$. 


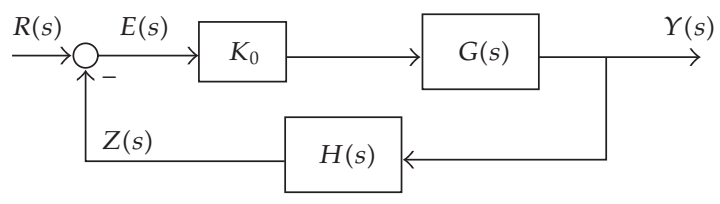

(a)

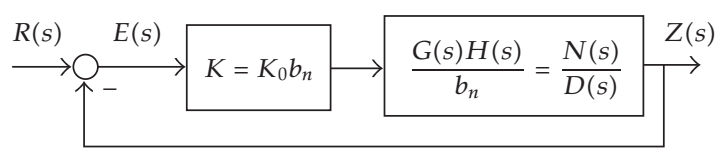

(b)

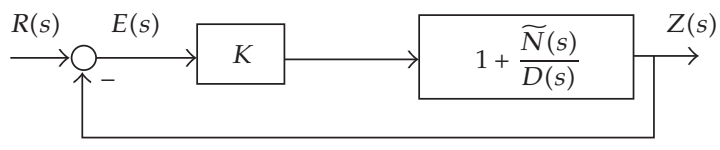

(c)

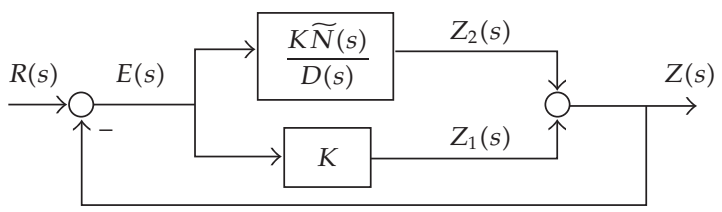

(d)

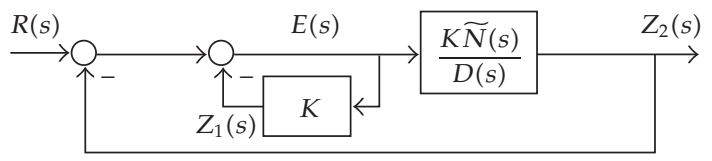

(e)

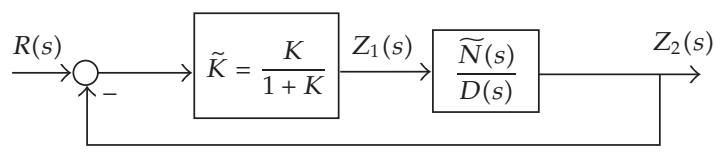

(f)

Figure 2: An alternative proof of Theorem 3.1: the block diagrams (a), (b), (c), (d), (e), and (f) are equivalent, for $K \neq-1$.

Remark 3.4. Figure 3 shows also that for $K \rightarrow-1,|\tilde{K}| \rightarrow \infty$. It means that in this situation, the root-loci plots of (3.3), for the open-loop transfer function $\widetilde{K} \widetilde{N}(s) / D(s)$, will present at least one asymptote, because it is a strictly proper transfer function. Therefore, at least one branch of these plots will approach infinity in the s-plane. It was firstly presented in [14] and the analysis above offers an alternative proof of this fact. The aforementioned gain $K=-1$ is equivalent to the case where one has the critical gain defined in [14]. Furthermore, from Figure 3 note that for $\widetilde{K} \rightarrow 1,|K| \rightarrow \infty$.

Remark 3.5. The characteristic equations (3.2) and (3.3) present the same open-loop poles, because for $K=0$ and $\widetilde{K}=0$ it follows from these equations that $D(s)=0$. Then, the procedure proposed in Theorem 3.1 preserves the open-loop poles of the original system, given in Figure 1. 


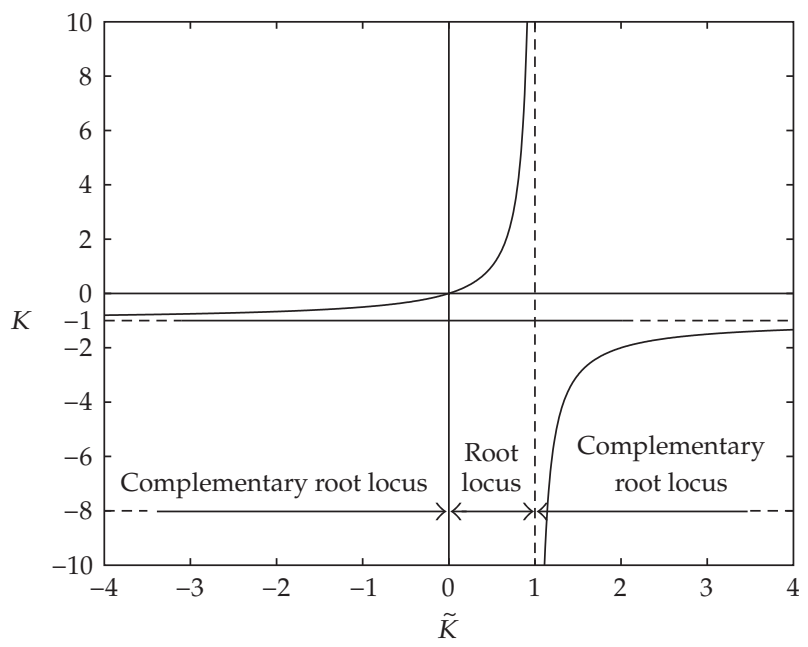

Figure 3: Relation between the gains $K$ and $\tilde{K}$, from (3.7): $K=\tilde{K} /(1-\tilde{K})$.

In order to exemplify the results of the above theorem, the next examples are presented.

Example 3.6. Consider the open-loop transfer function, that is, biproper, in Figure 1:

$$
K_{0} G(s) H(s)=K_{0} \frac{s^{2}+3 s-18}{s^{2}-4}
$$

In this case, $K=K_{0}, N(s)=s^{2}+3 s-18$, and $D(s)=s^{2}-4$. Then, from Theorem 3.1, and (3.5), $\widetilde{N}(s)=N(s)-D(s)=3 s-14$. Therefore, from (3.3) it follows that

$$
\widetilde{K} \widetilde{N}(s)+D(s)=\widetilde{K}(3 s-14)+\left(s^{2}-4\right)=0
$$

Note that (3.12) is equivalent to the characteristic equation $1+\widetilde{K} \widetilde{N}(s) / D(s)=0$, where $\widetilde{K} \widetilde{N}(s) / D(s)$ is a strictly proper transfer function.

It is easy to plot the root locus $(0 \leq \widetilde{K}<\infty)$ of (3.12). The open-loop poles and the zero are $p_{1}=-2$ e $p_{2}=2$ and $z_{1}=4.6667$, respectively. There exists one asymptote with angle equal to $180^{\circ}$ and the sections of the real axis that are occupied by this root locus are $2 \leq \operatorname{Re}(s) \leq 4.6667$ and $\operatorname{Re}(s) \leq-2$. Figure 4 shows this root-locus plot.

Now, the complementary root locus $(-\infty<\tilde{K} \leq 0)$ of (3.12) can be directly plotted using the construction rules presented in [17-21], because $\widetilde{K} \widetilde{N}(s) / D(s)$ is a strictly proper transfer function, and the problems with these rules, pointed out in [14], will not appear. Following these construction rules one can conclude that the open-loop poles and the zero are $p_{1}=-2$ e $p_{2}=2$ and $z_{1}=4.6667$, respectively. There exists one asymptote with angle equal to $0^{\circ}$, and the sections of the real axis that are occupied by this complementary root locus are $-2 \leq \operatorname{Re}(s) \leq 2$ and $\operatorname{Re}(s) \geq 4$.6667. Furthermore, the breakway points are equal to 0.4503 and 8.8830 , and there is only one point $(s=0)$ where the complementary root locus intersects the imaginary axis. Figure 5 shows this complementary root-locus plot. 


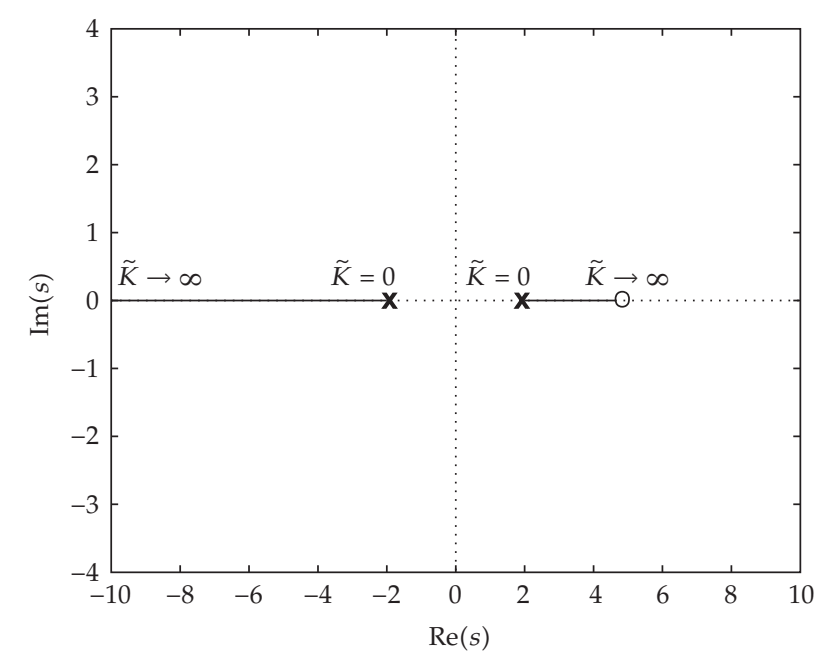

Figure 4: The root locus plot of Example 3.6 from (3.12).

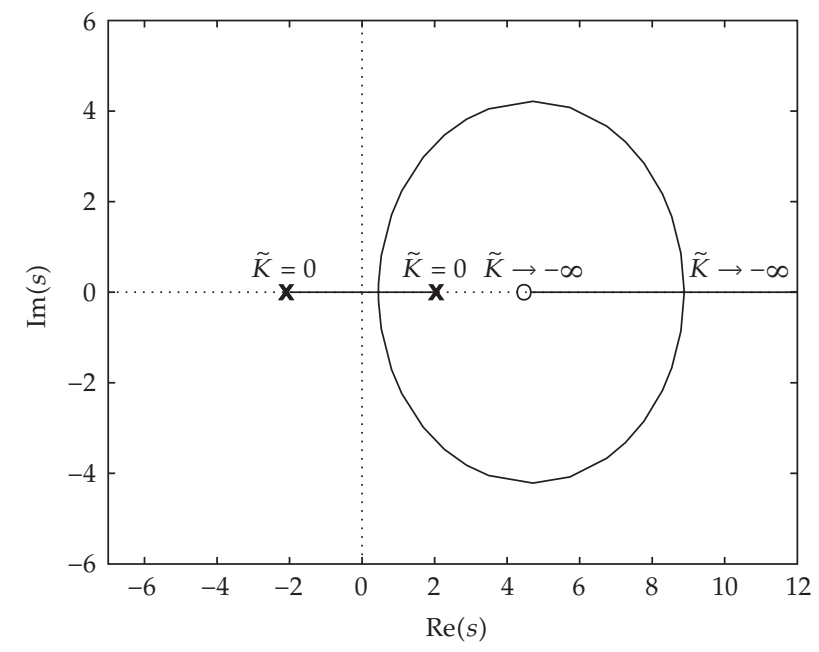

Figure 5: The complementary root locus plot from (3.12).

Another method to plot this complementary root locus, using only the root-locus construction rules, was proposed in [22]. Following this method, define $s=-v$. Therefore, one can get from (3.12)

$$
\widetilde{K} \widetilde{N}(-v)+D(-v)=\widetilde{K}(-3 v-14)+\left(v^{2}-4\right)=0
$$

Hence, defining $K_{p}=-\tilde{K}$ it follows that

$$
K_{p}(v+14)+\left(v^{2}-4\right)=0
$$




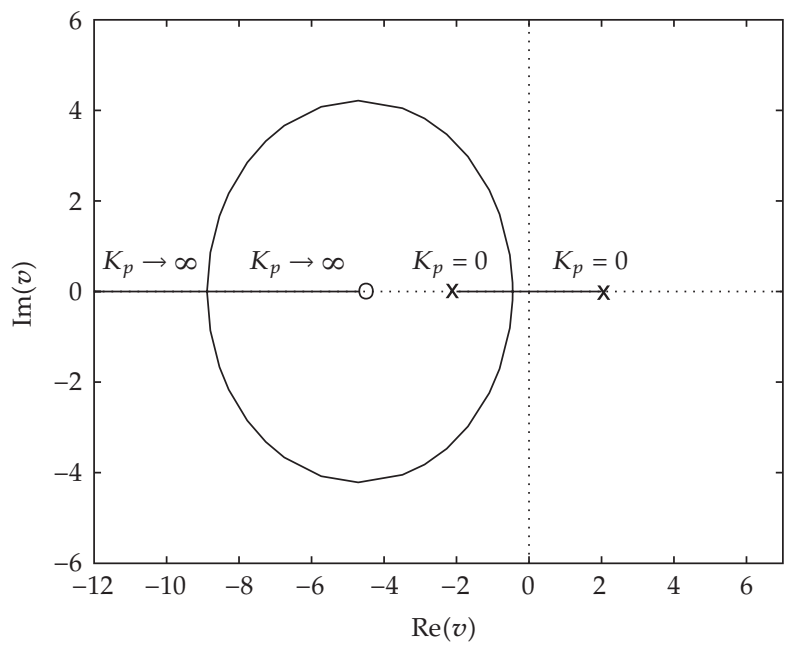

Figure 6: The root-locus plot in the plane $v$ from (3.14).

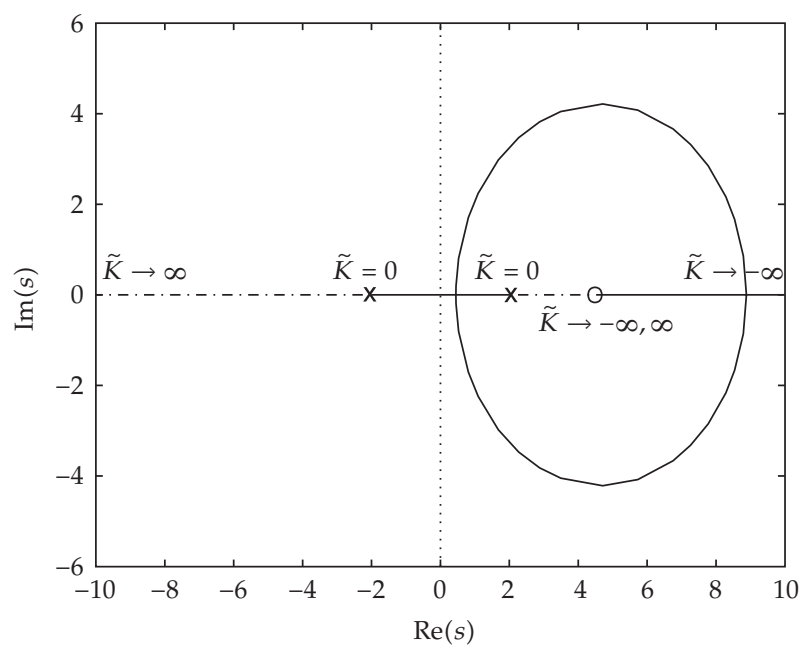

Figure 7: The complete root-locus plot of (3.12): root locus $(\cdot-\cdot)$ and complementary root locus $(-)$.

Now, we can easily plot the root locus of the above equation, in the complex plane $v$ and with the gain $0<K_{p}<\infty$. Figure 6 displays this plot. Finally, from the expressions $s=-v$ and $K=-K_{p}$, one can obtain the complementary root locus in the complex plane $s$ and with the gain $K$, by rotating $180^{\circ}$ the $v$ plane around the imaginary axis [22]. This plot is the same obtained with the complementary root-locus construction rules and it is also given in Figure 5.

The complete root-locus plot $(-\infty<\tilde{K}<\infty)$ of $(3.12)$ can be obtained from the plots of Figures 4 and 5 and is presented in Figure 7.

Now, from Figure 3 and (3.7) one can directly plot the root locus and complementary root locus with respect to the gain $K$. From Figure 3, the root locus $(0 \leq K<\infty)$ corresponds to the region $0 \leq \tilde{K}<1$. The closed-loop poles for $\widetilde{K}=1$, from (3.12) (or Figure 4 or Figure 7), are equal to 3 and -6 . This root-locus plot is shown in Figure 8 and it was directly obtained 


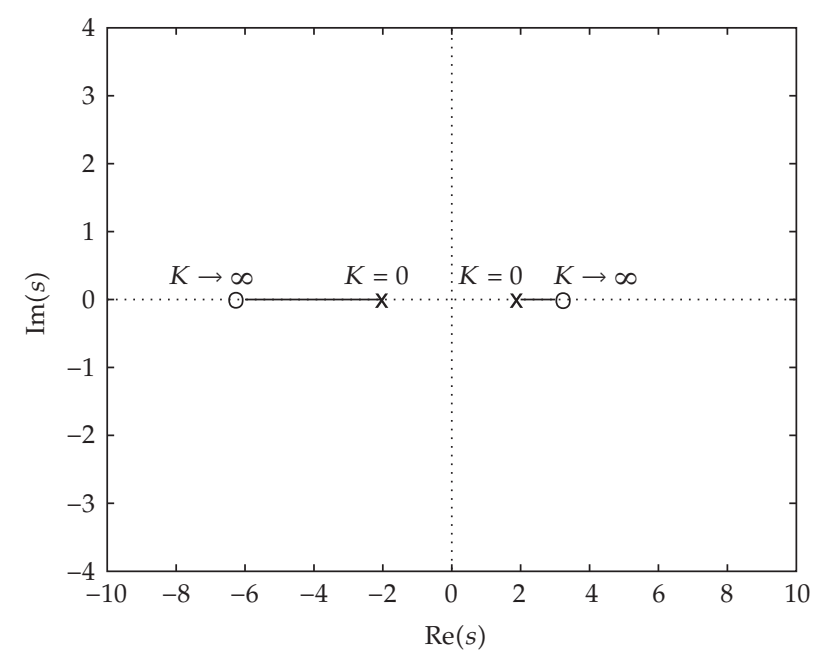

Figure 8: The root-locus plot of (3.11) for $K(0 \leq \tilde{K}<1)$.

from Figure 4 or Figure 7. Now, again from Figure 3, the complementary root locus $(-\infty<$ $K \leq 0)$ corresponds to the regions $-\infty<\tilde{K} \leq 0$ and $1<\tilde{K}<\infty$. Therefore, from Figure 7 one can get this complementary root locus, as described in Figure 9. For each gain $\tilde{K}$ in Figure 7 , the correspondent gain $K$ in Figure 8 and Figure 9 can be obtained using the expression given in (3.7). Finally, observe that the method proposed in [22] cannot be used to exactly plot the complementary root locus of (3.11). Thus, this example shows that the proposed method extends the results given in [22].

Remark 3.7. The root locus and complementary root locus plots presented in this paper, that illustrate the proposed method, were obtained by using the software MATLAB. The main contribution of this paper is an alternative analysis of the complementary root locus of biproper transfer functions, without using the theorems presented in [14].

Example 3.8. Consider the open-loop transfer function $K_{0} G(s) H(s)$ in Figure 1:

$$
K_{0} G(s) H(s)=K_{0} \frac{2 s^{4}+5 s^{3}+6 s^{2}+8 s+12}{3 s^{4}+8 s^{3}+9 s^{2}+12 s-16} .
$$

The open-loop poles are $p_{1}=-2.4643, p_{2}=0.6932$, and $p_{3,4}=-0.4478 \pm 1.7093 j$, and the zeros are $z_{1,2}=-1.5750 \pm 0.8068 j$, and $z_{3,4}=0.3250 \pm 1.3455 j$.

Eydgahi and Ghavamzadeh [14] have shown that the complementary root locus of this system presents a root at infinity. Then, considering that this system has the same number of poles and zeros, the authors concluded that some rules to plot complementary root locus are not valid in this case. In [14] this problem was studied, and new definition and construction rules for complementary root locus were proposed. In [22] it was proposed a method and its application to approximately plot this complementary root locus, using only the well-known root-locus construction rules. 


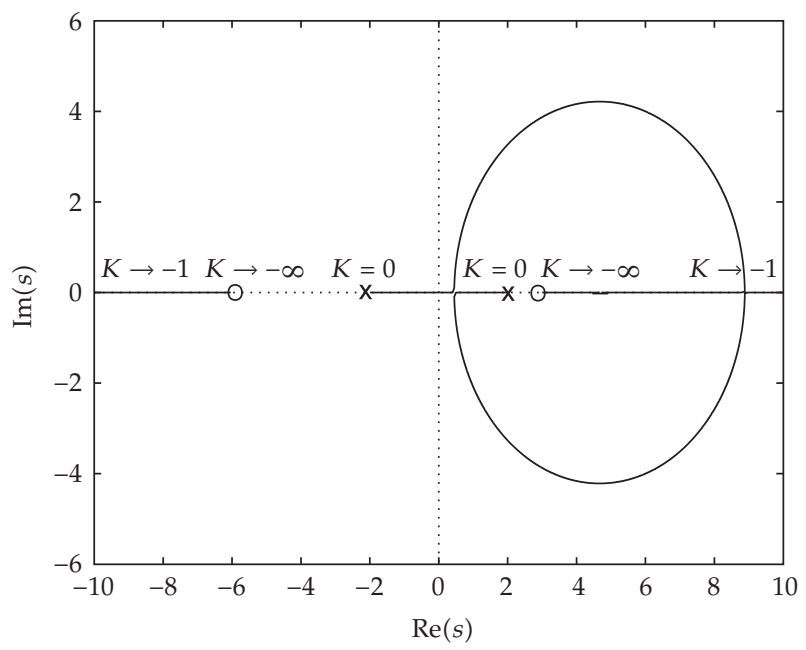

Figure 9: The complementary root-locus plot of (3.11) for $K(-\infty<\tilde{K} \leq 0$ and $1<\tilde{K}<\infty)$.

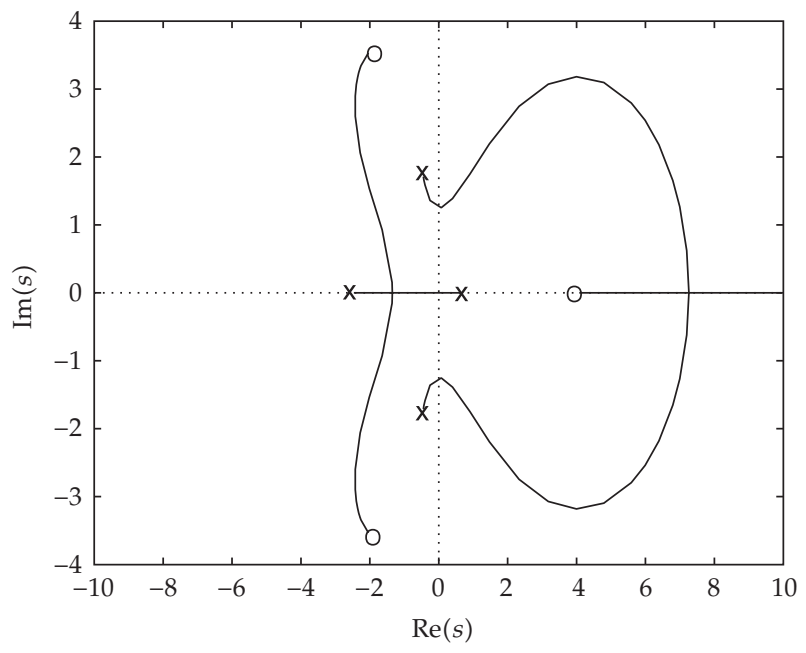

Figure 10: The root-locus plot from (3.17) with the gain $\tilde{K}$.

Now, one can use the results of Theorem 3.1 to exactly plot this complementary root locus. Following this procedure, from $(2.1)-(3.2)$ then $N(s)=s^{4}+2.5 s^{3}+3 s^{2}+4 s+6, D(s)=$ $s^{4}+2.6667 s^{3}+3 s^{2}+4 s-5.3333, K=2 K_{0} / 3$, and from (3.5) it follows that

$$
\widetilde{N}(s)=N(s)-D(s)=-0.1667 s^{3}+11.3333
$$

Therefore, from (3.3),

$$
\tilde{K} \widetilde{N}(s)+D(s)=\tilde{K}\left(-0.1667 s^{3}+11.3333\right)+\left(s^{4}+2.6667 s^{3}+3 s^{2}+4 s-5.3333\right)=0 .
$$




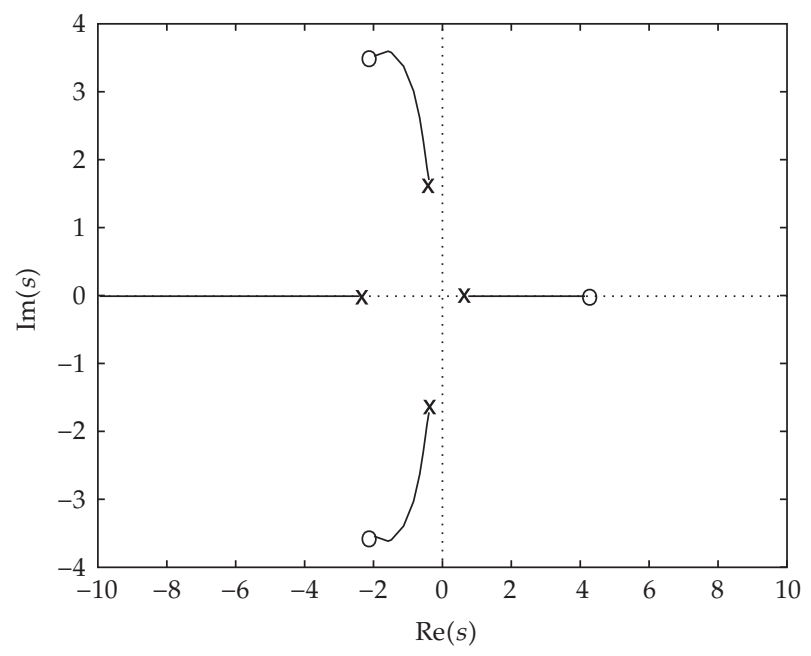

Figure 11: The complementary root-locus plot from (3.17) with the gain $\tilde{K}$.

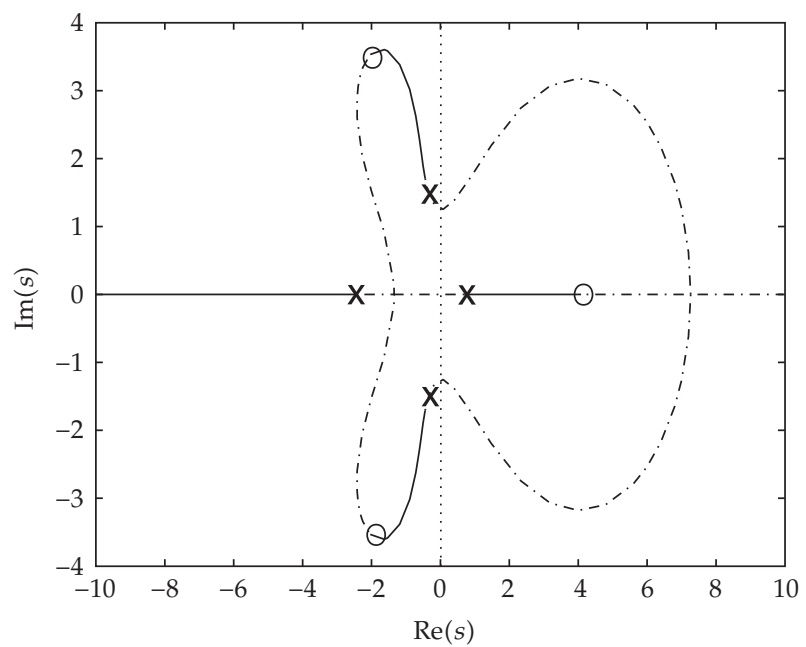

Figure 12: The complete root-locus plot from (3.17) with the gain $\tilde{K}$.

Note that (3.17) is equivalent to the characteristic equation $1+\widetilde{K} \widetilde{N}(s) / D(s)=0$, where $\widetilde{K} \widetilde{N}(s) / D(s)$ is a strictly proper transfer function. Therefore, one can plot the root locus $(0 \leq \widetilde{K}<\infty)$ and the complementary root locus $(-\infty<\tilde{K} \leq 0)$ of $(3.17)$, where the gain is $\widetilde{K}$, using the well-known construction rules [17-21]. These two plots are shown in Figures 10 and 11, respectively. The complete root locus $(-\infty<\tilde{K}<\infty)$ is given in Figure 12 .

Hence, from Figure 10 and (3.7), it is easy to obtain the root locus of (3.2), that corresponds to the regions where $0 \leq \widetilde{K}<1$ (see Figure 3 for more details). This plot is presented in Figure 13.

The complementary root locus of (3.2) can also be directly plotted from Figure 11, Figure 12 , and (3.7) for $-\infty<\tilde{K} \leq 0$ and $1<\tilde{K}<\infty$. Figure 14 shows this plot. 


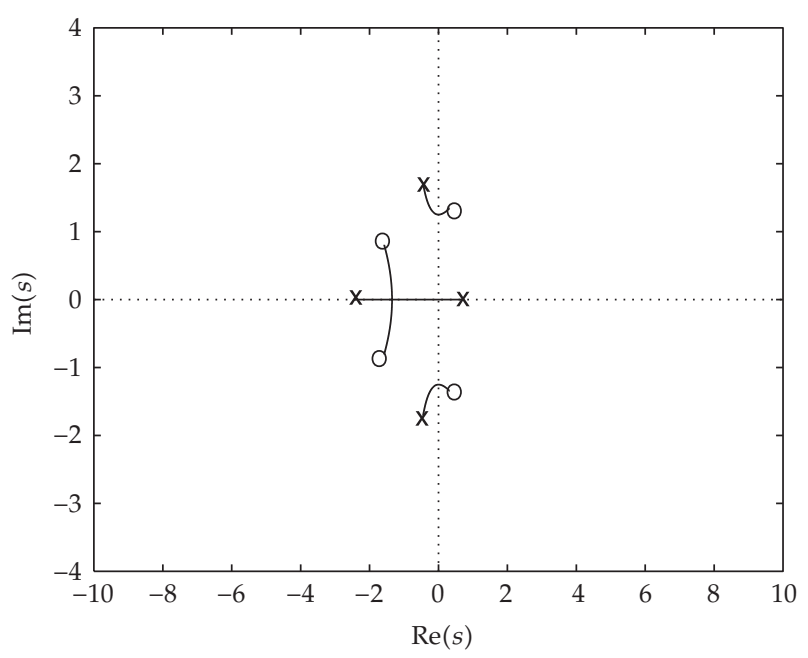

Figure 13: The root-locus plot from (3.2) with the gain $K$.

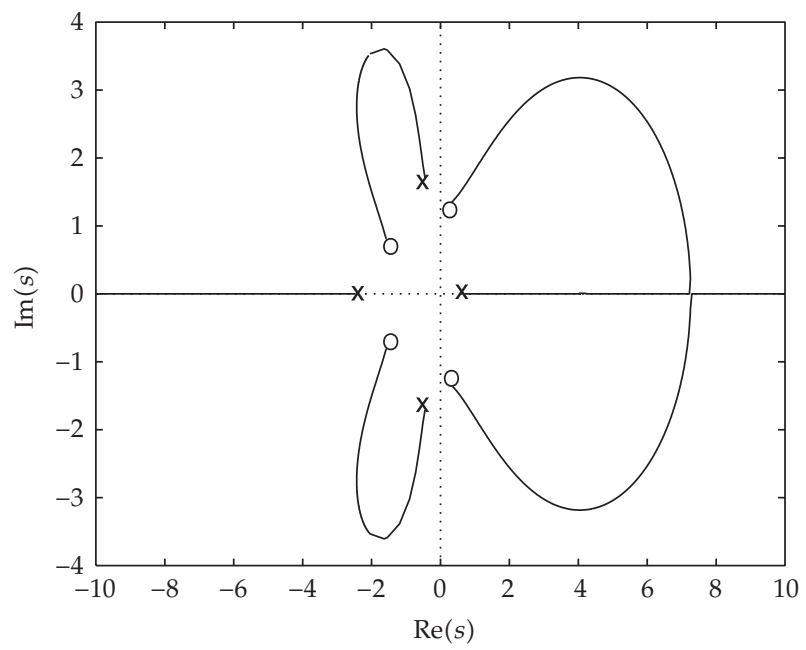

Figure 14: The complementary root-locus plot from (3.2), with the gain $K$.

\section{Conclusion}

A new method for plotting complementary root locus of biproper open-loop transfer functions has been presented in this article. The main idea was to show that this plot can be directly obtained from the root locus and complementary root locus of a suitable strictly proper transfer function. With this method it is not necessary to perform the additional analysis proposed in [14], that must complete the construction rules to plot complementary root locus.

The proposed method allows an exact plot of complementary root locus, of biproper open-loop transfer functions, using only well-known root locus rules. From the method presented in [22], for this case, one can only obtain an approximated plot. There exists a lack 
of sources about complementary root locus. This paper offers an alternative procedure and provides a better comprehension about this subject. Finally, the educators that teach block diagrams can also use the analysis depicted in Figure 2, as an interesting application of this theory.

\section{Acknowledgments}

The authors gratefully acknowledge the partial financial support by FAPESP, CAPES, and CNPq of Brazil.

\section{References}

[1] W. R. Evans, "Graphical analysis of control systems," Transactions of the American Institute of Electrical Engineers, vol. 67, no. 1, pp. 547-551, 1948.

[2] W. R. Evans, "Control system synthesis by root locus method," Transactions of the American Institute of Electrical Engineers, vol. 69, no. 1, pp. 66-69, 1950.

[3] F. Merrikh-Bayat and M. Afshar, "Extending the root-locus method to fractional-order systems," Journal of Applied Mathematics, vol. 2008, Article ID 528934, 13 pages, 2008.

[4] F. Merrikh-Bayat, M. Afshar, and M. Karimi-Ghartemani, "Extension of the root-locus method to a certain class of fractional-order systems," ISA Transactions, vol. 48, no. 1, pp. 48-53, 2009.

[5] D. L. Spencer, L. Philipp, and B. Philipp, "Root loci design using Dickson's technique," IEEE Transactions on Education, vol. 44, no. 2, pp. 176-184, 2001.

[6] M. C. M. Teixeira, “Direct expressions for Ogata's lead-lag design method using root locus," IEEE Transactions on Education, vol. 37, no. 1, pp. 63-64, 1994.

[7] M. C. M. Teixeira and E. Assunção, “On lag controllers: design and implementation," IEEE Transactions on Education, vol. 45, no. 3, pp. 285-288, 2002.

[8] M. C. M. Teixeira, E. Assunção, and M. R. Covacic, "Proportional controllers: direct method for stability analysis and MATLAB implementation," IEEE Transactions on Education, vol. 50, no. 1, pp. 74-78, 2007.

[9] J. R. C. Piqueira and L. H. A. Monteiro, "All-pole phase-locked loops: calculating lock-in range by using Evan's root-locus," International Journal of Control, vol. 79, no. 7, pp. 822-829, 2006.

[10] J. C. Basilio and S. R. Matos, "Design of PI and PID controllers with transient performance specification," IEEE Transactions on Education, vol. 45, no. 4, pp. 364-370, 2002.

[11] V. A. Oliveira, L. V. Cossi, M. C. M. Teixeira, and A. M. F. Silva, "Synthesis of PID controllers for a class of time delay systems," Automatica, vol. 45, no. 7, pp. 1778-1782, 2009.

[12] V. A. Oliveira, M. C. M. Teixeira, and L. Cossi, "Stabilizing a class of time delay systems using the Hermite-Biehler theorem," Linear Algebra and Its Applications, vol. 369, pp. 203-216, 2003.

[13] K. S. Narendra, "Inverse root locus, reversed root locus or complementary root locus?" IRE Transactions on Automatic Control, vol. 26, no. 3, pp. 359-360, 1961.

[14] A. M. Eydgahi and M. Ghavamzadeh, "Complementary root locus revisited," IEEE Transactions on Education, vol. 44, no. 2, pp. 137-143, 2001.

[15] C. T. Chen, Analog and Digital Control System Design, Saunders College, Orlando, Fla, USA, 1993.

[16] R. C. Dorf and R. H. Bishop, Modern Control Systems, Addison-Wesley, Reading, Mass, USA, 1998.

[17] J. J. D'Azzo and C. H. Houpis, Linear Control System Analysis and Design: Conventional and Modern, McGraw-Hill, New York, NY, USA, 1995.

[18] G. F. Franklin, J. D. Powell, and A. Emami-Naeini, Feedback Control of Dynamic Systems, AddisonWesley, Reading, Mass, USA, 1994.

[19] B. C. Kuo, Automatic Control Systems, Prentice-Hall, Englewood Cliffs, NJ, USA, 1995.

[20] K. Ogata, Modern Control Engineering, Prentice-Hall, Englewood Cliffs, NJ, USA, 1997.

[21] J. Rowland, Linear Control Systems: Modeling, Analysis, and Design, John Wiley \& Sons, New York, NY, USA, 1986. 
[22] M. C. M. Teixeira, E. Assunção, and E. R. M. D. Machado, "A method for plotting the complementary root locus using the root-locus (positive gain) rules," IEEE Transactions on Education, vol. 47, no. 3, pp. 405-409, 2004.

[23] L. H. A. Monteiro and J. J. Da Cruz, "Simple answers to usual questions about unusual forms of the Evans' root locus plot," Controle y Automação, vol. 19, no. 4, pp. 444-449, 2008.

[24] M. C. M. Teixeira, H. F. Marchesi, and E. Assunção, "Signal-flow graphs: direct method of reduction and MATLAB implementation," IEEE Transactions on Education, vol. 44, no. 2, pp. 185-190, 2001. 


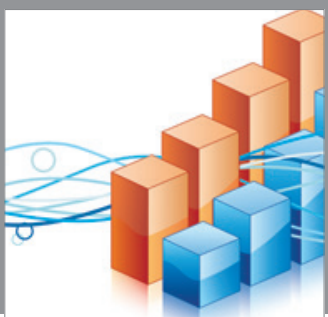

Advances in

Operations Research

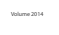

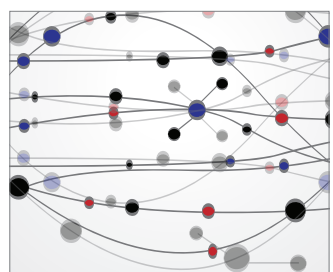

\section{The Scientific} World Journal
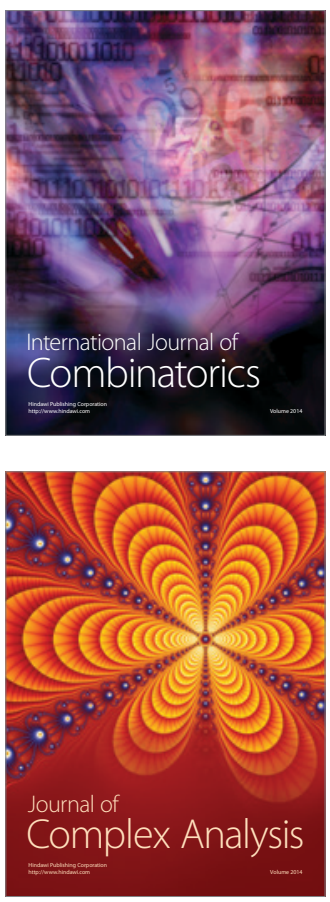

International Journal of

Mathematics and

Mathematical

Sciences
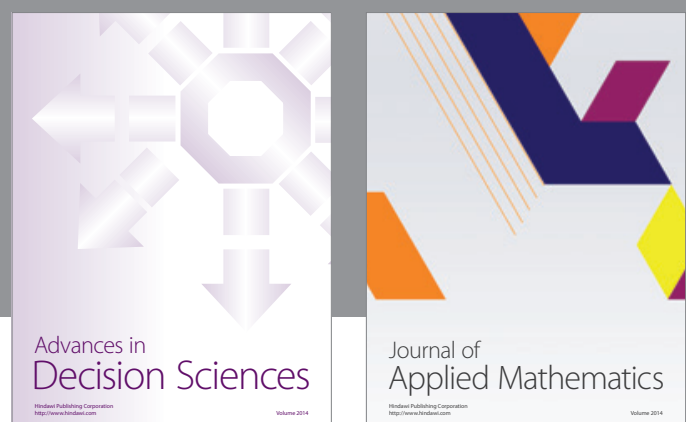

Journal of

Applied Mathematics
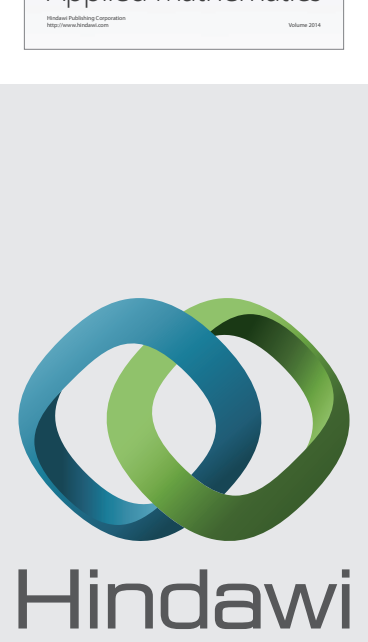

Submit your manuscripts at http://www.hindawi.com
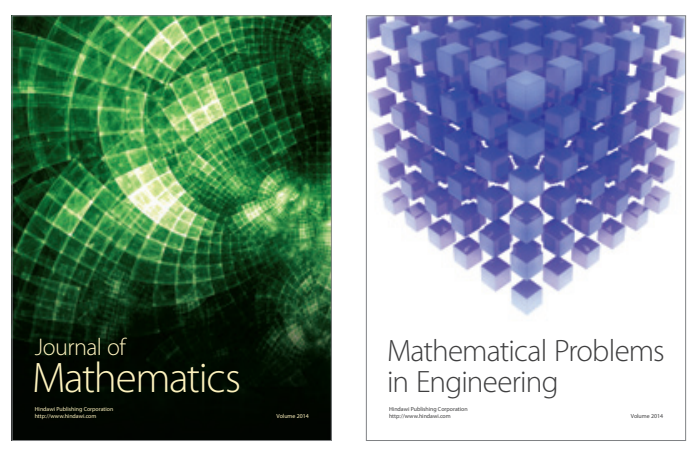

Mathematical Problems in Engineering
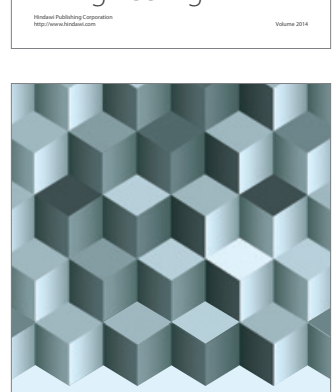

Journal of

Function Spaces
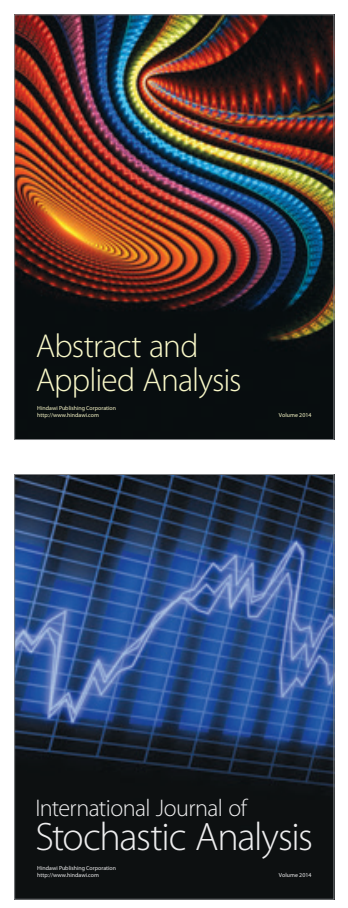

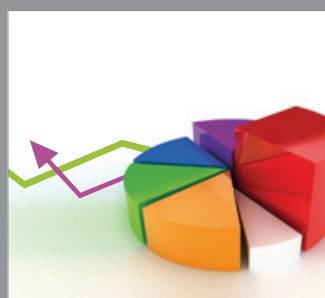

ournal of

Probability and Statistics

Promensencen
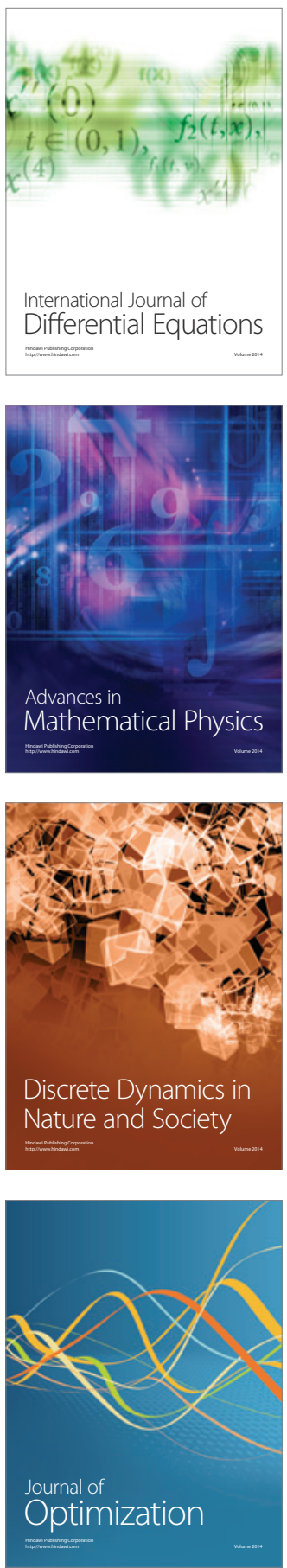